\title{
Light and Electron Microscopy of Microsporida using Enzyme Metallography
}

\author{
R. Powell,* V. Joshi, ${ }^{*}$ A. Thelian,* W. Liu,* P. Takvorian,** A. Cali,** and J. Hainfeld* \\ * Nanoprobes, Incorporated, 95 Horse Block Road, Yaphank, NY, 11980 \\ ** Rutgers University, Biological Sciences, 101 Warren Street, Newark, NJ 07102
}

Enzyme metallography (EnzMet $\left.{ }^{\mathrm{TM}}\right)$ is a biological labeling and staining method in which a targeted enzymatic probe is used to selectively deposit metal at sites of interest [1]. It has proven highly sensitive and specific for light microscopic applications such as in situ hybridization [2] and immunohistochemistry [3]. The granular staining shows that diffusion of the reaction product is negligible, providing higher resolution than most organic chromogens. The small size of the enzymatic probe and in situ metal deposition should afford improved access to interior or hindered antigens and denser labeling than larger colloidal gold probes, and with the high contrast provided by the deposited metal, make this a valuable immunoelectron microscopy method.

Enzyme metallography was evaluated for light and electron microscopy in Microsporida. These parasitic organisms are important opportunistic pathogens in AIDS and other immune compromised patients, where they are responsible for chronic diarrhea, malabsorption syndromes, myositis, and disseminating infections demonstrated in all tissues of the body. Approximately a dozen different microsporidia infect humans [4]. All form a diagnostic spore containing a coiled polar filament surrounding the single nucleus or paired abutted nuclei (diplokaryon) and its associated cytoplasmic organelles, the sporoplasm; upon germination, the polar filament is everted, becoming a tubule through which the spore contents travel to become the infective sporoplasm [5].

Cultured RK-13 cells infected with E. hellem microsporidia were grown on slides, immunofixed (for electron microscopy), and stored in PBS buffer. These were incubated with primary antibody (anti polar tube - PTP-55 [6], 1:100) for one hour. A universal detection system incorporating a biotinylated secondary antibody and polymerized peroxidase-streptavidin detection (I-View, Ventana Medical Systems) was then applied. After washing with PBS-0.01\% Tween-20, then distilled water, then $0.02 \mathrm{M}$ sodium citrate buffer at $\mathrm{pH} 3.8$, specimens were developed with a modified formulation of the enzyme metallographic reagent (Nanoprobes, Incorporated), washed with $0.02 \mathrm{M}$ sodium citrate buffer at $\mathrm{pH} 3.8$, rinsed with deionized water, and coverslipped. After light microscope examination, areas of interest were marked on the back of the slides. The cover glasses were removed, and the slides rinsed in distilled water, dehydrated through a series of ethanol solutions (50\% - 100\%) and infiltrated with Araldite 502 resin (EMS, PA) overnight. Marked areas were covered with BEEM capsules filled with resin, and embedded at $60^{\circ} \mathrm{C}$ for 24 hours. Thin sections were cut, placed on copper grids, and stained with uranyl acetate and lead citrate. Samples were examined using an FEI Tecnai 12 transmission electron microscope operated at $80 \mathrm{kV}$.

The polar tubes were easily observed with brightfield optics (arrows), and background was very clean: almost no non-specific deposits were observed on the cells and surrounding matrix. The enzyme metallography visualization is more sensitive, with lower background and higher resolution than DAB staining. This method offers the promise of both a superior research tool for ultrastructural examination, and a simplified light microscopic assay for Microsporidian infection.

References:

[1] J. F. Hainfeld, et al., Microsc. Microanal. 8 (Suppl. 2) (2002) 916 CD.

[2] R. Tubbs, J. Pettay, et al. J. Mol. Histol. 35 (2004) 589.

[3] R. Tubbs, J. Pettay, J., et al. Appl. Immunohistochem. Mol. Morphol. 13 (2005) 371. 
[4] A. Cali \& P. M. Takvorian, Southeast Asian Trop. Med. Public Health, 35 (Suppl. 1) (2004) 58.

[5] A. Cali, L. M. Weiss, and P. M. Takvorian, J. Euk. Microbiol., 49 (2003) 164.

[6] E.M. Keohane, G.A. Orr, et al., Mol. Biochem. Parasitol., 94 (1998) 227.

[7] This work was supported by NIH SBIR grant 2R44 GM064257-02A1 and NIH grant 2R01 AI031788. The authors thank Ventana Medical Systems, Incorporated for the I-View reagent.

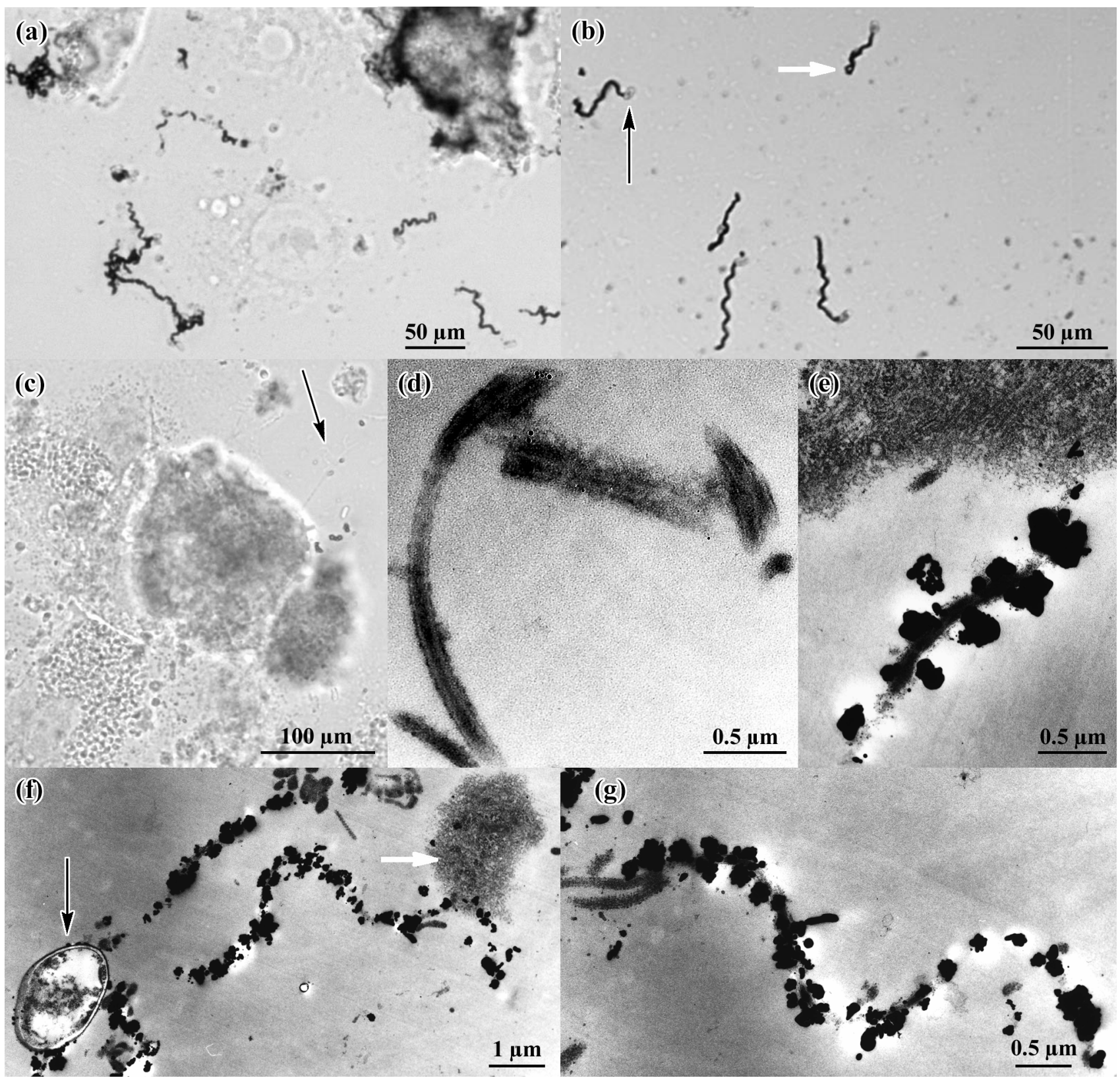

Figures: RK-13 cells infected with E. hellem microsporidia, stained using anti-polar tube antibody (PTP-55) and I-View universal peroxidase detection system developed with enzyme metallography. Brightfield light microscopy: (a) 40X objective; (b) 40X oil immersion objective, showing infective sporoplasm (dark arrow) and empty spore (white arrow); (c) control with primary antibody omitted, 20X objective; arrow indicates unstained polar tube. TEM: (d) control with primary antibody omitted, showing counterstained polar tube; (e) - (g) staining with primary antibody, showing heavily decorated polar tube, empty spore (dark arrow) and infective sporoplasm (white arrow). 